\title{
SUBGROUPS OF TWISTED WREATH PRODUCTS
}

\author{
PÉTER P. PÁLFY \\ Alfréd Rényi Institute of Mathematics, Hungarian Academy of Sciences \\ Reáltanoda utca 13-15, 1053 Budapest, Hungary \\ Email: ppp@renyi.hu
}

\begin{abstract}
By determining subdirect products invariant under the action of a regular permutation group of the components we provide a natural motivation for the definition of twisted wreath products. Then - based on papers of R. Baddeley, A. Lucchini, F. Börner, and M. Aschbacher - we explain how twisted wreath products play a fundamental role in the problem of representing finite lattices as intervals in subgroup lattices of finite groups.
\end{abstract}

\section{Introduction}

Our first goal is to provide a natural motivation for the definition of twisted wreath products. Originally, the twisted wreath product was introduced by Bernhard H. Neumann [17] in 1963. At first glance his definition looks quite complicated. Michio Suzuki [22, Chapter 2, §10] presented a more elegant treatment of this construction. In Section 2 we will determine all those subdirect products in a direct product of isomorphic non-abelian simple groups that are invariant under a regular permutation group of the components. This naturally leads to the definition of the twisted wreath product.

Twisted wreath products occur in the O'Nan-Scott-Aschbacher Theorem on the classification of primitive finite permutation groups. They were erroneously omitted from the first version [20] of the theorem, and were only added later to the list in the paper of Michael Aschbacher and Leonard Scott [5], and independently by László Kovács [14]. (See also [16].)

Although in the original paper of B. H. Neumann [17], as well as in several later developments, twisted wreath products were used for the construction of infinite groups with certain peculiar properties, in the present paper we will restrict our attention to twisted wreath products of finite groups.

Our second goal is to explain the role twisted wreath products play for the problem of representing finite lattices as intervals in subgroup lattices of finite groups. This was explicitly or implicitly observed in the papers of Robert Baddeley and Andrea Lucchini [7], Baddeley [6], Ferdinand Börner [8], and Michael Aschbacher [2]. Based on their results we present in Section 3 a simplified proof showing that this representation problem can be reduced either to the case of almost simple groups or to the case of twisted wreath products. In Section 4 we give proper credits to the original papers and make further comments on the related literature. 
We tried to make the paper as much self-contained as possible. However, at several places the proof makes use of Schreier's Hypothesis claiming that the outer automorphism group $\operatorname{Out}(T)=\operatorname{Aut}(T) / \operatorname{Inn}(T)$ of every finite non-abelian simple group $T$ is solvable. This is a well-known consequence of the classification of finite simple groups.

As for many questions in finite group theory it would be desirable to reduce the problem to the case of almost simple groups (groups $G$ with a simple normal subgroup $T$ with $\mathbf{C}_{G}(T)=1$ ). However, it seems inevitable to consider also certain twisted wreath products in the context of representing finite lattices as intervals in subgroup lattices of finite groups.

The basic group theoretic notions do not need explanation for the readership of this proceedings. As lattice theory is concerned, let us recall that a lattice $L$ is a partially ordered set where any two elements $x, y$ have a greatest lower bound (called their meet, denoted by $x \wedge y$ ) and a least upper bound (their join, denoted by $x \vee y$ ). Finite lattices have a smallest and a largest element; these will be denoted by $0_{L}$ and $1_{L}$. By a filter $F$ in a finite lattice $L$ we mean a non-empty subset of the form $\{x \in L \mid x \geq a\}$ for some $a \in L$. We obtain the dual of a lattice when we reverse the ordering, so the meet in the dual lattice is the same as the join in the original lattice, and similarly, the new join is the old meet.

A lattice $L$ is called modular if $\forall x, y, z \in L: x \leq z \Rightarrow(x \vee y) \wedge z=x \vee(y \wedge z)$. The subgroup lattice of an abelian group is always modular. We will call a lattice $L$ consisting of more than two elements strongly non-modular if for every $y \in L$, $y \neq 0_{L}, 1_{L}$, there exists a pair of elements $x<z \in L$ such that $y \vee x=y \vee z$ and $y \wedge x=y \wedge z$.

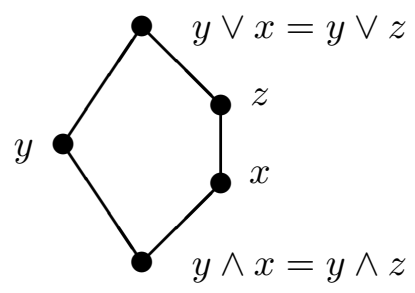

\section{Invariant subdirect products}

Let $T$ (the "target") be a finite non-abelian simple group and let $D$ (the "domain") be an arbitrary finite group. Consider the group $F$ of all functions $D \rightarrow T$ with pointwise multiplication. Then $F \cong T \times \cdots \times T=T^{|D|}$. Let $D$ act on $F$ by translation, that is for $f \in F, d \in D$ let $f^{d} \in F$ be the function

$$
f^{d}(x)=f\left(x d^{-1}\right) \quad(x \in D) .
$$

Clearly, this defines an action of the group $D$ on $F$, as we have $f^{d_{1} d_{2}}(x)=$ $f\left(x\left(d_{1} d_{2}\right)^{-1}\right)=f\left(\left(x d_{2}^{-1}\right) d_{1}^{-1}\right)=f^{d_{1}}\left(x d_{2}^{-1}\right)=\left(f^{d_{1}}\right)^{d_{2}}(x)$. The semidirect product $F \rtimes D$ is the regular wreath product $T$ ? $D$.

Recall that a subgroup $H \leq G_{1} \times \cdots \times G_{n}$ of a direct product is said to be a subdirect product, if the projection of $H$ to each factor $G_{i}(i=1, \ldots, n)$ is 
surjective. If the factors are pairwise isomorphic non-abelian simple groups, then the structure of any subdirect product is described by the following lemma (see, e.g., [9, Exercise 4.3]).

Lemma 2.1 Let $T$ be a non-abelian simple group and let $H \leq T^{n}$ be a subdirect product. Then $H \cong T^{m}$ for some $1 \leq m \leq n$. Moreover, there exist a map $\nu:\{1, \ldots, n\} \rightarrow\{1, \ldots, m\}$ and automorphisms $\varphi_{i} \in \operatorname{Aut}(T)$ such that $f:\{1, \ldots, n\} \rightarrow T$ belongs to $H$ iff $f(i)=\varphi_{i}\left(t_{\nu(i)}\right)$ with $t_{1}, \ldots, t_{m} \in T$.

Now we are going to determine which subdirect products in $F \cong T \times \cdots \times T$ are invariant under the action of $D$. Let $H \leq F$ be a subdirect product. By the lemma we have $H \cong T^{m}$ for some $1 \leq m \leq|D|$, and

$$
H=\left\{f: D \rightarrow T \mid f(x)=\varphi_{x}\left(t_{\nu(x)}\right), t_{1}, \ldots, t_{m} \in T\right\},
$$

with appropriate $\nu: D \rightarrow\{1, \ldots, m\}$ and $\varphi_{x} \in \operatorname{Aut}(T)(x \in D)$. For $f \in H$, $b \in D$, the invariance of $H$ means that $f^{b^{-1}}$ also belongs to $H$, hence with some $u_{1}, \ldots, u_{m} \in T$ we have

$$
f^{b^{-1}}(x)=\varphi_{x}\left(u_{\nu(x)}\right)
$$

By the definition of the action of $D$ we obtain

$$
f^{b^{-1}}(x)=f(x b)=\varphi_{x b}\left(t_{\nu(x b)}\right) .
$$

Clearly, $D$ preserves the partition given by the kernel of the map $\nu$, hence it is a partition into the cosets of some subgroup $D_{0} \leq D: D=D_{0} x_{1} \cup D_{0} x_{2} \cup \cdots \cup D_{0} x_{m}$ with $\nu(d)=i$ iff $d \in D_{0} x_{i}$. Without loss of generality we may assume that $x_{1}=1$ and that for every $i=1, \ldots, m$ we have $\varphi_{x_{i}}=$ id. If $b \in D_{0} x_{i}$ and $a \in D_{0}$, then $a b \in$ $D_{0} x_{i}$ as well. Then $f(a b)=f^{b^{-1}}(a)=\varphi_{a}\left(u_{\nu(a)}\right)=\varphi_{a}\left(u_{1}\right)$. For $a=1$ this yields $u_{1}=f(b)$, hence $\forall a \in D_{0}, \forall b \in D: f(a b)=\varphi_{a}(f(b))$. If $b=1$ we get $\forall a \in D_{0}$ : $f(a)=\varphi_{a}(f(1))$. If $a, b \in D_{0}$, then $\varphi_{a b}(f(1))=f(a b)=\varphi_{a}(f(b))=\varphi_{a}\left(\varphi_{b}(f(1))\right)$. Since $H$ is a subdirect product, $f(1)$ can be any element of $T$, so $\varphi: D_{0} \rightarrow \operatorname{Aut}(T)$ is a homomorphism. Furthermore, $\varphi_{a x_{i}}\left(t_{i}\right)=f\left(a x_{i}\right)=\varphi_{a}\left(f\left(x_{i}\right)\right)=\varphi_{a}\left(t_{i}\right)$, so $\forall a \in D_{0}, \forall i \in\{1, \ldots, m\}: \varphi_{a x_{i}}=\varphi_{a}$.

Conversely, it is easy to verify that if $D_{0} \leq D, D=D_{0} x_{1} \cup \cdots \cup D_{0} x_{m}$, and $\varphi: D_{0} \rightarrow \operatorname{Aut}(T)$ is a homomorphism, then by defining

$$
\operatorname{Sdp}\left(D_{0}, \varphi\right)=\left\{f: D \rightarrow T \mid f\left(a x_{i}\right)=\varphi_{a}\left(t_{i}\right), a \in D_{0}, t_{i} \in T(i=1, \ldots, m)\right\}
$$

we obtain a $D$-invariant subdirect product in $F \cong T^{|D|}$. Indeed, if $b \in D$, then multiplication by $b$ from the right permutes the right cosets of $D_{0}$ and so we have $x_{i} b=a_{i} x_{j}$ with $a_{i} \in D_{0}, 1 \leq j=j(i, b) \leq m$. If $f \in \operatorname{Sdp}\left(D_{0}, \varphi\right)$ let us denote $f\left(x_{i} b\right)$ by $u_{i}$. With this notation we have $f^{b^{-1}}\left(a x_{i}\right)=f\left(a x_{i} b\right)=f\left(a a_{i} x_{j}\right)=\varphi_{a a_{i}}\left(t_{j}\right)=$ $\varphi_{a}\left(\varphi_{a_{i}}\left(t_{j}\right)\right)=\varphi_{a}\left(f\left(a_{i} x_{j}\right)\right)=\varphi_{a}\left(f\left(x_{i} b\right)\right)=\varphi_{a}\left(u_{i}\right)$, so $f^{b^{-1}} \in \operatorname{Sdp}\left(D_{0}, \varphi\right)$, as we wanted.

Thus we have proved the following proposition. 
Proposition 2.2 If $T$ is a non-abelian simple group, $D$ is any finite group, and $F=\{f: D \rightarrow T\}$, then the D-invariant subdirect products in $F \cong T^{|D|}$ are precisely the subgroups of the form $\operatorname{Sdp}\left(D_{0}, \varphi\right)$ for subgroups $D_{0} \leq D$ and homomorphisms $\varphi: D_{0} \rightarrow \operatorname{Aut}(T)$.

We can define $\operatorname{Sdp}\left(D_{0}, \varphi\right)$ even without assuming the simplicity of $T$, the construction makes sense for any $T$. Then the semidirect product $\operatorname{Sdp}\left(D_{0}, \varphi\right) \rtimes D$ is the twisted wreath product $\operatorname{Twr}\left(T, D, D_{0}, \varphi\right)$ of $T$ and $D$ with respect to the subgroup $D_{0} \leq D$ and the homomorphism $\varphi: D_{0} \rightarrow \operatorname{Aut}(T)$.

If we want to compare invariant subdirect products, the following is obvious.

Lemma 2.3 A subdirect product $\operatorname{Sdp}\left(D_{1}, \varphi_{1}\right)$ is contained in another subdirect product $\operatorname{Sdp}\left(D_{2}, \varphi_{2}\right)$ iff $D_{1}$ contains $D_{2}$ and $\varphi_{2}$ is the restriction of $\varphi_{1}$ to $D_{2}$.

Now we return to analyzing the subgroup structure of $F$ when $T$ is a non-abelian simple group.

Lemma 2.4 If $T$ is a non-abelian simple group, and $D_{0} \leq D, \varphi: D_{0} \rightarrow \operatorname{Aut}(T)$ satisfy $\varphi\left(D_{0}\right) \geq \operatorname{Inn}(T)$, then every non-trivial $D$-invariant subgroup of $\operatorname{Sdp}\left(D_{0}, \varphi\right)$ is a subdirect product.

Proof. Let $H \leq \operatorname{Sdp}\left(D_{0}, \varphi\right)$ be any $D$-invariant subgroup. Consider the image $U=\{f(1) \mid f \in H\}$ of $H$ under the projection to the first coordinate. Let $u \in U$ (so $u=f(1)$ for some $f \in H$ ), and take an $a \in D_{0}$. Then $\varphi_{a}(u)=\varphi_{a}(f(1))=$ $f(a)=f^{a^{-1}}(1) \in U$, hence $U$ is a $\varphi\left(D_{0}\right)$-invariant subgroup of the simple group $T$. By assumption, $\varphi\left(D_{0}\right) \geq \operatorname{Inn}(T)$, so $U$ is a normal subgroup of $T$, hence by the simplicity of $T$, either $U=1$ or $U=T$. Since $D$ acts transitively on the components of the direct product, either all projections of $H$ have trivial image, and so $H=1$, or all projections map onto $T$, and so $H$ is a subdirect product.

Thus the $D$-invariant subgroups of $\operatorname{Sdp}\left(D_{0}, \varphi\right)$ apart from the trivial subgroup are all of the form $\operatorname{Sdp}\left(D_{1}, \varphi_{1}\right)$ with $D_{0} \leq D_{1}$ and $\left.\varphi_{1}\right|_{D_{0}}=\varphi$. Combining the previous two lemmas, we obtain the following.

Proposition 2.5 Let $T$ be a non-abelian simple group, and assume that $D_{0} \leq$ $D, \varphi: D_{0} \rightarrow \operatorname{Aut}(T)$ satisfy $\varphi\left(D_{0}\right) \geq \operatorname{Inn}(T)$. Then the lattice of $D$-invariant subgroups of $\operatorname{Sdp}\left(D_{0}, \varphi\right)$ is isomorphic to the dual of the lattice of all extensions of $\varphi$ to subgroups containing $D_{0}$, together with an additional top element (what corresponds to the trivial subgroup via the dual isomorphism).

Remark 2.6 Note that in the setting of (2.5) if $\varphi_{1}: D_{1} \rightarrow \operatorname{Aut}(T)$ extends $\varphi: D_{0} \rightarrow \operatorname{Aut}(T)$, then $\varphi_{1}$ is uniquely determined by its kernel. Indeed, if $K=$ $\operatorname{ker}\left(\varphi_{1}\right)$, then $D_{1} / K \cong \varphi_{1}\left(D_{1}\right) \leq \operatorname{Aut}(T)$ is an almost simple group, and the image of $D_{0}$ contains $\operatorname{Inn}(T) \cong T$, hence the action of $D_{1} / K$ on $\varphi^{-1}(\operatorname{Inn}(T)) / K \cong T$ determines the homomorphism $\varphi_{1}: D_{1} \rightarrow \operatorname{Aut}(T)$. 
Example 2.7 Let $A_{5}$ and $S_{5}$ denote the alternating and the symmetric group of degree 5 , and let $T=A_{5}, D=S_{5} \times A_{5}, D_{0}=\operatorname{diag}\left(A_{5}\right)=\left\{(x, x) \mid x \in A_{5}\right\}<D$, and $\varphi: D_{0} \cong A_{5} \rightarrow \operatorname{Aut}(T) \cong S_{5}$ an embedding. It is easy to see that the subgroups of $D$ containing $D_{0}$ are $D_{0}=\operatorname{diag}\left(A_{5}\right), A_{5} \times A_{5}$, and $D=S_{5} \times A_{5}$. Now $\varphi$ has two extensions to $A_{5} \times A_{5}$, corresponding to the first and the second projection. Likewise, there are two extensions to $S_{5} \times A_{5}$. Together with the additional top element this gives a hexagon lattice (here $a, b \in A_{5}, s \in S_{5}$ ):

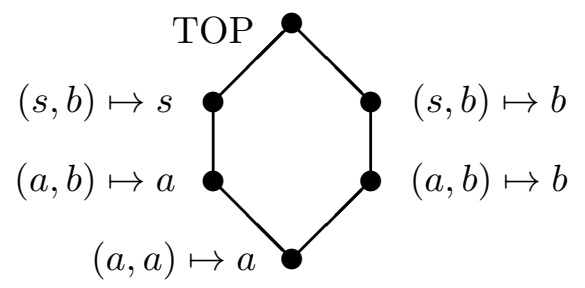

Hence by (2.5) the lattice of $D$-invariant subgroups of $\operatorname{Sdp}\left(D_{0} . \varphi\right)$ is the hexagon lattice.

If $M$ is a minimal normal subgroup of a finite group, then $M$ is characteristically simple, so it is either an elementary abelian $p$-group for some prime number $p$, or it is isomorphic to a direct power of a non-abelian simple group $T$. We consider the latter case, when $M=T_{1} \times \cdots \times T_{k}(k \geq 1)$ and each $T_{i} \cong T$. Now let a group $A$ act on $T_{1} \times \cdots \times T_{k}$ in such a way that $A$ permutes the direct factors transitively. Let $A_{1}=\left\{a \in A \mid T_{1}^{a}=T_{1}\right\}$ and denote by $\alpha$ the homomorphism $A_{1} \rightarrow \operatorname{Aut}\left(T_{1}\right)$ determined by the action of $A_{1}$. Furthermore, choose a set of coset representatives $x_{1}=1, x_{2}, \ldots, x_{k}$ of $A_{1}$ in $A$ and fix the isomorphism $t \mapsto t^{x_{i}}\left(t \in T_{1}\right)$ between $T_{1}=T$ and $T_{i}(i=1, \ldots, k)$. Then $\operatorname{Sdp}\left(A_{1}, \alpha\right) \cong T^{k}$ with the isomorphism given by the projection onto the group of functions $\left\{f:\left\{x_{1}, \ldots, x_{k}\right\} \rightarrow T\right\}$ and this isomorphism is compatible with the action of $A$. In the rest of the paper we will freely use this identification of $\operatorname{Sdp}\left(A_{1}, \alpha\right)$ and $T^{k}$. (Cf. [15].) The following lemmas will be needed in the proof of the main result (3.2).

Lemma 2.8 Every A-invariant subgroup of $T^{k}$ is one of the following types:

1. a subdirect product in $T^{k}$;

2. a box, that is, $U^{k}$ for some $A_{1}$-invariant subgroup $1<U<T$;

3. a skew subgroup, i.e., a nontrivial subgroup properly contained in a box;

4. the trivial subgroup.

Proof. Let $H \leq T^{k}$. Let the projection to the first component map $H$ onto $U \leq$ $T$. Since $H$ is $A$-invariant, each projection maps $H$ onto $U$ in the corresponding component (isomorphic to $T$ via the fixed isomorphism). So $H \leq U^{k}$. If $U=T$, then $H$ is a subdirect product. If $1<U<T$ and $H=U^{k}$, then $H$ is a box. If $1<U<T$ and $H<U^{k}$, then $H$ is a skew subgroup. (Note that the box $U^{k}$ is also $A$-invariant in this case.) If $U=1$, then $H=1$ as well. 
Corollary 2.9 A maximal A-invariant subgroup is either a subdirect product or a box, unless the only proper A-invariant subgroup is the trivial one.

The following is obvious.

Lemma 2.10 The box subgroups in $\mathrm{Sub}_{A}\left(T^{k}\right)$ form a sublattice isomorphic to $\operatorname{Sub}_{A_{1}}(T)$.

The next result is a well-known consequence of Schreier's Hypothesis, see [6, Proposition 2.4].

Lemma 2.11 If $\alpha\left(A_{1}\right) \nsupseteq \operatorname{Inn}(T)$, then there exists a proper non-trivial $A_{1}$-invariant subgroup of $T$.

Lemma 2.12 A box cannot be contained in a proper subdirect product.

Proof. For any proper subdirect product $H$ there exist a pair of indices $1 \leq i<$ $j \leq k$ and $\varphi \in \operatorname{Aut}(T)$ such that for any $\left(x_{1}, \ldots, x_{k}\right) \in H$ we have $x_{j}=\varphi\left(x_{i}\right)$. Hence if $x_{i}=1$, then $x_{j}=1$ as well. In the contrary, any box subgroup contains elements with $x_{i}=1, x_{j} \neq 1$.

Lemma 2.13 Let $H_{1}>H_{2}$ be A-invariant subdirect products and let $U$ be an $A_{1}$-invariant subgroup. Then either $H_{1} \cap U^{k}>H_{2} \cap U^{k}$ or $H_{2} \cap U^{k}=1$.

Proof. Let $H_{2}=\operatorname{Sdp}\left(B_{2}, \beta\right)$ and $H_{1}=\operatorname{Sdp}\left(B_{1},\left.\beta\right|_{B_{1}}\right)$ with $B_{1}<B_{2}$ (see (2.3)). Suppose that $H_{2} \cap U^{k} \neq 1$. By the definition of $H_{2}=\operatorname{Sdp}\left(B_{2}, \beta\right)$ this means that there exists $t_{1}, \ldots, t_{m} \in T\left(m=\left|A: B_{2}\right|\right)$ not all equal to 1 , such that $\beta_{b}\left(t_{i}\right) \in U$ for every $b \in B_{2}$ and each $i=1, \ldots, m$. In particular, we can choose $1 \neq u \in U$ such that $\beta_{b}(u) \in U$ for every $b \in B_{2}$. Then the function $f: A \rightarrow T$ given by $f(a)=\beta_{a}(u)$, if $a \in B_{1}$, and $f(a)=1$, otherwise, belongs to $H_{1}=\operatorname{Sdp}\left(B_{1},\left.\beta\right|_{B_{1}}\right)$, but not to $H_{2}$, hence $H_{1} \cap U^{k}>H_{2} \cap U^{k}$.

\section{Intervals in subgroup lattices of finite groups}

Let $\operatorname{Sub}(G)$ denote the subgroup lattice of the group $G$, and for a subgroup $H<G$ we denote by $\operatorname{Int}(H, G)=\{X \mid H \leq X \leq G\}$ the lattice of intermediate subgroups (in other words: overgroups of $H$ ), and call it the interval between $H$ and $G$ in the subgroup lattice. If a group $A$ acts by automorphisms on $G$, then the $A$-invariant subgroups form a sublattice in $\operatorname{Sub}(G)$; it will be denoted by $\operatorname{Sub}_{A}(G)$. Likewise, we will use the notation $\operatorname{Int}_{A}(H, G)$, whenever $H$ is an $A$-invariant subgroup of $G$.

Intervals of subgroup lattices occur in various contexts. If $F \subset E$ is a finite separable field extension and $E^{*}$ is the splitting field containing $E$, then the lattice of intermediate fields $\{X \mid F \subseteq X \subseteq E\}$ is dually isomorphic to the interval $\operatorname{Int}\left(\operatorname{Gal}\left(E^{*} \mid E\right), \operatorname{Gal}\left(E^{*} \mid F\right)\right)$ in the Galois group of $E^{*}$.

In the theory of operator algebras it is an open problem whether every finite lattice is isomorphic to the lattice of intermediate subfactors of a von Neumann algebra. Yasuo Watatani [24] proved that whenever a lattice can be represented as 
an interval in a subgroup lattice of a finite group, then it also occurs as a lattice of intermediate subfactors of a von Neumann algebra. With the exception of two lattices, he was able to find intervals isomorphic to every lattice with at most six elements. One of the missing cases was the hexagon lattice. M. Aschbacher [2] gave a general construction whose particular cases provided examples for the hexagon and for the other six-element lattice Watatani was not able to handle.

In universal algebra a well-known open problem asks whether every finite lattice is isomorphic to the congruence lattice of a finite algebra. This problem is motivated by the fundamental result of George Grätzer and E. Tamás Schmidt [11] stating that every algebraic lattice is isomorphic to the congruence lattice of some algebraic structure. (A lattice is called algebraic iff it is complete and every element is a join of compact elements. In particular, every finite lattice is algebraic.) For almost all finite lattices each known proof of the Grätzer-Schmidt Theorem constructs infinite algebras to represent the lattice as a congruence lattice. So it is a natural question to ask, if finite algebras with prescribed finite congruence lattice can be constructed. In a joint paper with Pavel Pudlák [19] we proved that the problem about general algebraic structures is actually equivalent to a group theoretic problem.

Problem 3.1 Is every finite lattice isomorphic to an interval in the subgroup lattice of a finite group?

One direction of the equivalence is obvious. Let $G$ act on the set of right cosets of the subgroup $H$, and consider each permutation in $G$ as an operation with one variable. Then the congruences are exactly the partitions into cosets of subgroups belonging to the interval $\operatorname{Int}(H, G)$, hence the congruence lattice of this multiunary algebra is isomorphic to this interval. Concerning the reverse implication, it should be emphasized that we do not claim that the congruence lattices of finite algebras are (up to isomorphism) the same as the intervals in subgroup lattices of finite groups. What we proved is that if all finite lattices can be represented as congruence lattices of finite algebras then all finite lattices can be represented as intervals in subgroup lattices of finite groups. In fact, we embed any finite lattice into a finite lattice with some useful properties, and then we show that the smallest algebra with a congruence lattice having these properties is a transitive permutation group considered as a multi-unary algebra.

It was shown by Jiří Tůma [23] that every algebraic lattice is isomorphic to an interval in the subgroup lattice of an infinite group. So it is the finiteness of the group what seems to constitute a severe restriction. Therefore, it is generally believed that the answer to the finite representation problem is negative.

Making use of ideas from the fundamental papers of R. Baddeley and A. Lucchini [7], R. Baddeley [6], F. Börner [8], and M. Aschbacher [2] we present here a simplified proof for a slightly modified version of the main result of F. Börner [8] giving a reduction of the problem to almost simple groups and to twisted wreath products.

Theorem 3.2 Every finite lattice is isomorphic to an interval in the subgroup lattice of a finite group if and only if one of the following is true: 
(1) Every finite lattice consisting of more than one element is isomorphic to an interval $\operatorname{Int}(H, G)$ in the subgroup lattice of an almost simple finite group $G$ with a core-free subgroup $H$ (that is, $\bigcap_{g \in G} g^{-1} H g=1$ ).

(2) Every finite lattice consisting of more than one element is isomorphic to an interval $\operatorname{Int}(D, G)$ in the subgroup lattice of a twisted wreath product $G=$ $\operatorname{Twr}\left(T, D, D_{0}, \varphi\right)$ of a non-abelian finite simple group $T$ and a finite group $D$ with respect to a subgroup $D_{0}<D$ and a homomorphism $\varphi: D_{0} \rightarrow \operatorname{Aut}(T)$ satisfying $\varphi\left(D_{0}\right) \geq \operatorname{Inn}(T)$.

In the proof we will make use of the following lemmas.

Lemma 3.3 Every finite lattice $L$ can be embedded as a filter into a finite lattice $\hat{L}$ generated by its coatoms (maximal elements in $\hat{L} \backslash\left\{1_{\hat{L}}\right\}$ ).

Proof. We define $\hat{L}=L \cup\left\{c_{x}^{1}, c_{x}^{2}, a_{x} \mid x \in L \backslash\left\{0_{L}, 1_{L}\right\}\right\} \cup\left\{c^{11}, c^{12}, c^{21}, c^{22}, a^{1}, a^{2}, 0^{*}\right\}$ and we extend the order on $L$ to $\hat{L}$ in the following way: each new element is $<1_{L}$; $a_{x}<y$ if $x \leq y$ in $L ; a^{1}, a^{2}<y$ for all $y \in L ; 0^{*}$ is smaller than every other element of $\hat{L} ; a_{x}<c_{x}^{1}, c_{2}^{2}, a^{1}<c^{11}, c^{12}, a^{2}<c^{21}, c^{22}$. It is straightforward to check that $\hat{L}$ is a lattice and $L$ is the filter in $\hat{L}$ consisting of the elements above $0_{L}$. Moreover, the elements $c_{x}^{1}, c_{x}^{2}, c^{11}, c^{12}, c^{21}, c^{22}$ are coatoms and they generate the whole lattice $\hat{L}$, since $a_{x}=c_{x}^{1} \wedge c_{x}^{2}, a^{1}=c^{11} \wedge c^{12}, a^{2}=c^{21} \wedge c^{22}, 0^{*}=a^{1} \wedge a^{2}, 0_{L}=a^{1} \vee a^{2}$, $x=a_{x} \vee 0_{L}\left(x \in L \backslash\left\{0_{L}, 1_{L}\right\}\right)$, and $1_{L}=c^{11} \vee c^{12}$.

Lemma 3.4 If $N \triangleleft G$ and $N \leq H$, then $\operatorname{Int}(H, G) \cong \operatorname{Int}(H / N, G / N)$.

Lemma 3.5 If $N \triangleleft G, H<G$ with $N H=G$, then $\operatorname{Int}(H, G) \cong \operatorname{Int}_{H}(H \cap N, N)$.

Proof. It is easy to check that the maps $U \mapsto U \cap N(U \in \operatorname{Int}(H, G))$ and $V \mapsto V H$ $\left(V \in \operatorname{Int}_{H}(H \cap N, N)\right)$ are order-preserving, and are inverses to each other, showing the isomorphism of the two intervals.

Lemma 3.6 Assume that $\operatorname{Int}(H, G)$ is a strongly non-modular lattice. Then for any normal subgroup $N \triangleleft G$ either $N \leq H$ or $N H=G$. Moreover, if $H$ is core-free, then $G$ has a unique minimal normal subgroup $M$ and $M$ is not abelian.

Proof. Suppose that $H<N H<G$. Since the interval is strongly non-modular, there exist subgroups $H<X<Z<G$ with $N H \wedge X=N H \wedge Z$ and $N H \vee X=$ $N H \vee Z$. Now $N X$ is a subgroup and $N X=N \vee X=N \vee H \vee X=N H \vee X=$ $N H \vee Z \geq Z$, hence every $z \in Z$ can be written as $z=n x$ with $n \in N$ and $x \in X$. Then $n=z x^{-1} \in N H \wedge Z=N H \wedge X \leq X$, so $z=n x \in X$, that is $Z \leq X$, a contradiction.

Suppose now that $H$ is core-free, and let $M \triangleleft G$ be a minimal normal subgroup. Then $M H=G$, and by (3.5) $\operatorname{Int}(H, G) \cong \operatorname{Int}_{H}(H \cap M, M)$. This lattice is not modular, hence $M$ cannot be abelian. If $M^{*}$ is another minimal normal subgroup, then $M$ and $M^{*}$ elementwise commute, so any $H$-invariant subgroup of $M$ is also $M^{*} H$-invariant, i.e., normal in $G$ (since $M^{*} H=G$ ). Then the minimality of $M$ gives that $\operatorname{Int}_{H}(H \cap M, M)$ can have at most two elements, although we have 
included in the definition of strongly non-modular lattices that they have more than two elements.

Proof of the Theorem. We have to prove that if both (1) and (2) fail, then there exists a finite lattice that cannot be represented as an interval in the subgroup lattice of a finite group. Let $L_{1}$ be a lattice that is not isomorphic to $\operatorname{Int}(H, G)$ in the subgroup lattice of any finite almost simple group $G$ with a core-free subgroup $H$, and let $L_{2}$ be a lattice that is not isomorphic to $\operatorname{Int}(D, G)$ in the subgroup lattice of any twisted wreath product $G=\operatorname{Twr}\left(T, D, D_{0}, \varphi\right)$ of a non-abelian finite simple group $T$ and a finite group $D$ with respect to a subgroup $D_{0}<D$ and a homomorphism $\varphi: D_{0} \rightarrow \operatorname{Aut}(T)$ satisfying $\varphi\left(D_{0}\right) \geq \operatorname{Inn}(T)$. Let us embed $L_{1}$ as a filter into a finite lattice $\hat{L}_{1}$ that is generated by its coatoms (see (3.3)). Now let $L$ be the following lattice assembled together using the parts $\hat{L}_{1}, L_{2}$, their duals $\hat{L}_{1}^{d}, L_{2}^{d}$, and two hexagons:

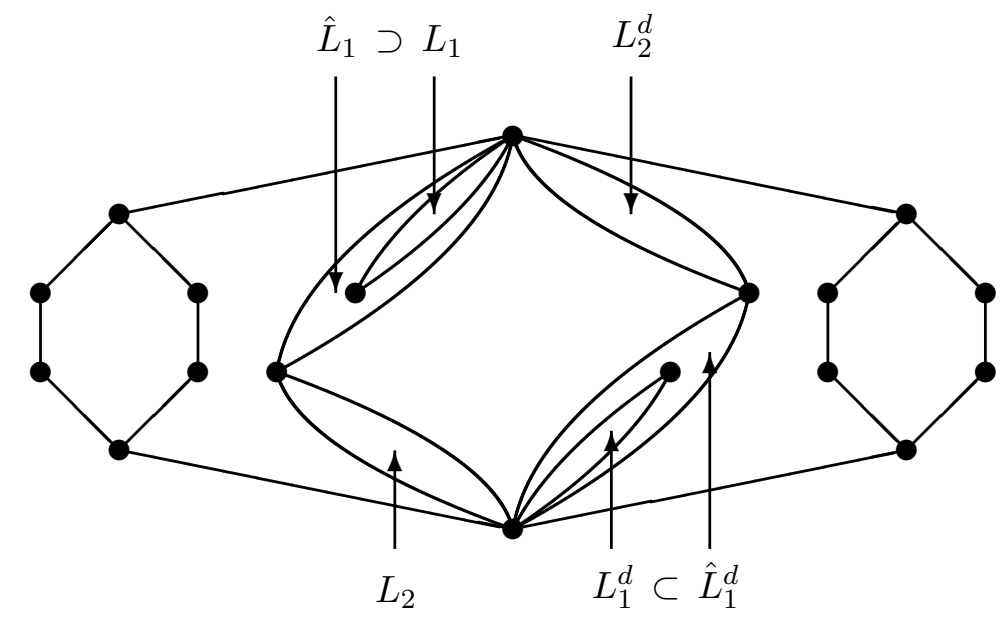

Notice that $L$ is a self-dual lattice. It is also easy to see that $L$ is a strongly non-modular lattice. We will show that our assumptions on $L_{1}$ and $L_{2}$ imply that $L$ cannot be isomorphic to an interval in the subgroup lattice of any finite group. Suppose the contrary, that there exist a finite group $G$ and a subgroup $H<G$ such that $\operatorname{Int}(H, G) \cong L$. We assume that $G$ has minimal order among groups with an interval isomorphic to $L$ in $\operatorname{Sub}(G)$.

Clearly, $H$ is a core-free subgroup in $G$ (cf. (3.4)). Let $M$ be a minimal normal subgroup of $G$. Since the interval $\operatorname{Int}(H, G)$ is a strongly non-modular lattice, (3.6) implies that $M H=G, M$ is the unique minimal normal subgroup in $G$, and $M=T_{1} \times \cdots \times T_{k}(k \geq 1)$ with pairwise isomorphic non-abelian simple groups $T_{1}, \ldots, T_{k}$. Now $H$ permutes the direct factors of $M$ transitively. Let $H_{1}=\mathbf{N}_{H}\left(T_{1}\right)$ and let $\alpha: H_{1} \rightarrow \operatorname{Aut}\left(T_{1}\right)$ give the automorphisms of $T_{1}$ induced by conjugation by elements of $H_{1}$. Furthermore, take coset representatives $x_{1}=1, x_{2}, \ldots, x_{k}$ of $H_{1}$ in $H$ so that $x_{i}^{-1} T_{1} x_{i}=T_{i}(i=1, \ldots, k)$. For each $i$ fix the isomorphism $t \mapsto x_{i}^{-1} t x_{i}$ $\left(t \in T_{1}\right)$ between $T_{1}$ and $T_{i}$, so that $M$ becomes $T^{k}$ (where $T=T_{1}$ ). As in Section 2 we may identify $M$ with $\operatorname{Sdp}\left(H_{1}, \alpha\right) \leq T^{|H|}$. Now $\operatorname{Int}(H, G) \cong \operatorname{Int}_{H}(H \cap M, M)$ (see (3.5)). 
Step 1. We show that $H \cap M=1$.

Following (2.8) we distinguish four cases for the subgroup $H \cap M$ :

1. $H \cap M$ is a subdirect product in $M=T^{k}$;

2. $H \cap M$ is a box, i.e., $H \cap M=U^{k}$ for some $H_{1}$-invariant subgroup $1<U<T$;

3. $H \cap M$ is a skew subgroup, i.e., a nontrivial subgroup properly contained in a box;

4. $H \cap M=1$.

If $H \cap M$ is a subdirect product, then every subgroup containing it is also a subdirect product. In virtue of (2.2) let $H \cap M$ be determined by a subgroup $H_{0} \leq H$ containing $H_{1}$ and a homomorphism $\beta: H_{0} \rightarrow \operatorname{Aut}(T)$ extending $\alpha$. Then (2.5) implies that $\operatorname{Int}_{H}(H \cap M, M)$ is isomorphic to the dual of the interval $\operatorname{Int}\left(H_{1}, H_{0}\right)$, so this latter lattice is also isomorphic to $L$, contrary the minimal choice of $G$.

If $H \cap M=U^{k}$ is a box, then it follows from (2.9) and (2.12) that all maximal invariant subgroups in $\operatorname{Int}_{H}(H \cap M, M)$, i.e., all coatoms are also boxes. Since $\hat{L}_{1}$ is generated by coatoms, we obtain that all invariant subgroups in this subinterval are boxes, hence $\hat{L}_{1} \cong \operatorname{Int}_{H_{1}}(V, T)$ for an appropriate $H_{1}$-invariant subgroup $V$. As $L_{1}$ is a filter in $\hat{L}_{1}$, we obtain that $L_{1}$ occurs as an interval in the subgroup lattice of the almost simple group $T H_{1} / \mathbf{C}_{T H_{1}}(T)$, contrary to our assumption on $L_{1}$.

If $H \cap M$ is a skew subgroup, then let $U^{k}$ be the box containing it, i.e., the direct product of the images of the projections of $H \cap M$. Then $H \cap M<U^{k}<M=T^{k}$, and at least one of the hexagons $\operatorname{in}_{\operatorname{Int}_{H}}(H \cap M, M)$, say, $\operatorname{Int}(X, Z)$ (where $X<$ $\left.Z \in \operatorname{Int}_{H}(H \cap M, M)\right)$ does not contain $U^{k}$. The top element of the hexagon, $Z$ is a maximal $H$-invariant subgroup in $T^{k}$, hence it is either a subdirect product or a box (see (2.9)). If it were a box, then $Z \cap U^{k}=H \cap M$ would be a box as well, which is not the case. So $Z$ is a subdirect product, and then (2.12) implies that $X$ is also a subdirect product. In this case, however, $Z \cap U^{k}=X \cap U^{k}=H \cap M \neq 1$ cannot hold by (2.13).

We conclude that $H \cap M=1$, so $G=M H$ is a semidirect product, in fact, it is the twisted wreath product $\operatorname{Twr}\left(T, H, H_{1}, \alpha\right)$.

Step 2. We exclude the possibility that the top elements of both hexagons in $\operatorname{Int}_{H}(1, M)$ are boxes.

Assume that these coatoms of $L$ correspond to the subgroups $U_{1}^{k}$ and $U_{2}^{k}$. Then $U_{1}$ and $U_{2}$ are maximal $H_{1}$-invariant subgroups of $T$. Since the normalizer of a $H_{1^{-}}$ invariant subgroup is also $H_{1}$-invariant and $T$ is a simple group, it follows that $U_{1}$ and $U_{2}$ are self-normalizing. As $U_{1}^{k} \cap U_{2}^{k}=H \cap M=1$, we get $\mathbf{N}_{T}\left(U_{1}\right) \cap \mathbf{N}_{T}\left(U_{2}\right)=1$. Therefore, if $h \in H_{1}$ induces an inner automorphism on $T$, then it is the trivial automorphism, that is, $\alpha\left(H_{1}\right) \cap \operatorname{Inn}(T)=1$.

We distinguish two cases, whether $\alpha\left(H_{1}\right)=1$ or not, and show that in both cases at least one of the subgroups $U_{1}, U_{2}$ is a $p$-group for some prime number $p$. If $\alpha\left(H_{1}\right)=1$, then all subgroups of $T$ are $H_{1}$-invariant. Now $U_{1}$ is a $p$-group, otherwise two Sylow subgroups corresponding to different prime divisors of $\left|U_{1}\right|$ would provide two $H$-invariant subgroups of $U_{1}^{k}$ with trivial intersection, which is 
not the case as $U_{1}^{k}$ is the top element of one of the hexagons in $L$. Now consider the case when $\alpha\left(H_{1}\right)$ is non-trivial. We have seen that $\alpha\left(H_{1}\right) \cap \operatorname{Inn}(T)=1$, hence $\alpha\left(H_{1}\right)$ is isomorphic to a subgroup of $\operatorname{Out}(T)$, which is a solvable group according to Schreier's Hypothesis. Let $A$ be a minimal normal subgroup in $\alpha\left(H_{1}\right) \leq \operatorname{Aut}(T)$. This is an elementary abelian $q$-group for some prime $q$. Now $\mathbf{C}_{T}(A)<T$ is a proper $H_{1}$-invariant subgroup. (It may be the trivial subgroup.) We can choose one of $i \in\{1,2\}$ so that the $H$-invariant box subgroup $\mathbf{C}_{T}(A)^{k}$ intersects $U_{i}^{k}$ trivially. Then we have $\mathbf{C}_{T}(A) \cap U_{i}=1$, that is, $A$ acts fixed point freely on $U_{i}$. It follows that $q$ does not divide $\left|U_{i}\right|$, and for every prime divisor $p$ of $\left|U_{i}\right|$ there is a unique $A$-invariant Sylow $p$-subgroup of $T$ (see $[1,18.7]$ ). The uniqueness implies that this Sylow subgroup is also $H_{1}$-invariant. It follows, as before, that $U_{i}$ is a $p$-group.

We have shown that in both cases $U_{i}(i=1$ or 2$)$ is a $p$-group. Observe that the Frattini subgroup $\Phi\left(U_{i}^{k}\right)$ is also $H$-invariant, as it is a characteristic subgroup of $U_{i}^{k}$. Since $U_{i}^{k} / \Phi\left(U_{i}^{k}\right)$ is abelian, the interval $\operatorname{Int}_{H}\left(\Phi\left(U_{i}^{k}\right), U_{i}^{k}\right)$ is a modular lattice consisting of at least two elements. Moreover, by the basic property of the Frattini subgroup, if $W \vee \Phi\left(U_{i}^{k}\right)=U_{i}^{k}$ for some subgroup $W$, then $W=U_{i}^{k}$. However, there is no element in the lattice $L$ that has these properties required from $\Phi\left(U_{i}^{k}\right)$. This contradiction shows that it is not possible that the top elements of both hexagons are boxes.

Step 3. Conclusion of the proof.

Now we may assume that the top element of one of the hexagons $\operatorname{in} \operatorname{Int}_{H}(1, M) \cong$ $L$ is a subdirect product. Let this hexagon be $\operatorname{Int}(X, Z)$. By (2.12) we see that $X$ is also a subdirect product. Hence (2.2) and (2.3) yield that $X=\operatorname{Sdp}\left(H_{X}, \beta\right)$ for a subgroup $H_{X} \leq H$ and a homomorphism $\beta: H_{X} \rightarrow \operatorname{Aut}(T)$, and the subgroups of $M$ containing $X$ are exactly the subdirect products $\operatorname{Sdp}\left(W,\left.\beta\right|_{W}\right)$ corresponding to subgroups $W \in \operatorname{Int}\left(H_{1}, H_{X}\right)$. (In particular, we have $\alpha=\left.\beta\right|_{H_{1}}$.) Let $Z=\operatorname{Sdp}\left(H_{Z},\left.\beta\right|_{H_{Z}}\right) \cong T^{\left|H: H_{Z}\right|}$. Since $\operatorname{Int}_{H}(1, Z)$ does not contain any box or skew subgroup, we must have $\beta\left(H_{Z}\right) \geq \operatorname{Inn}(T)$ (see (2.11)). Let $K$ be the kernel of $\beta$. Since $\operatorname{Int}\left(H_{Z}, H_{X}\right)$ is a hexagon, and that is a strongly non-modular lattice, it follows from (3.6) that either $K \leq H_{Z}$ or $K H_{Z}=H_{X}$. In the first case $\operatorname{Inn}(T) \leq \beta\left(H_{Z}\right) \cong H_{Z} / K<H_{X} / K \cong \beta\left(H_{X}\right) \leq \operatorname{Aut}(T)$, so the hexagon $\operatorname{Int}\left(H_{Z}, H_{X}\right) \cong \operatorname{Int}\left(\beta\left(H_{Z}\right) / \operatorname{Inn}(T), \beta\left(H_{X}\right) / \operatorname{Inn}(T)\right)$ is an interval in the subgroup lattice of the outer automorphism group of $T$. However, the outer automorphism group is solvable by Schreier's Hypothesis, but the subgroup lattice of a solvable group cannot contain a hexagon as an interval by (3.6). Thus we have $K H_{Z}=H_{X}$, and so $K H_{1}=H_{X}$ as well, thus $\beta\left(H_{1}\right)=\beta\left(H_{Z}\right) \geq \operatorname{Inn}(T)$. By (2.4) this means that with the exception of the trivial subgroup, $\operatorname{Int}_{H}(1, M)$ consists of subdirect products only. In particular, all elements apart from the trivial subgroup in the interval corresponding to $L_{2}$ are subdirect products, hence $L_{2}$ occurs as an interval in the subgroup lattice of a twisted wreath product as in (2), contrary to our assumption. This finishes the proof of Theorem 3.2. 


\section{Comments on related literature}

The definition of the twisted wreath product is given in the books by Bertram Huppert [13] and by Michio Suzuki [22]. Huppert [13, Definition I.15.10] gives the same definition as in the original paper of B. H. Neumann [17], and uses the German name verschränktes Kranzprodukt. Suzuki's definition [22, Chapter 2, Definition 10.3] is essentially the same as we have formulated it in Section 2. It is worth mentioning the analogy between twisted wreath products and induced representations, noticed by Dan Haran [12, Section 1].

The problem of representing finite lattices as intervals in subgroup lattices has raised considerable interest. For a survey see [18]. Although a negative answer is expected, only some deep reduction theorems and solutions for particular classes of lattices have been achieved so far. Recently Michael Aschbacher devoted several voluminous works to this problem. Here we can mention only two of these: one dealing with overgroups of root subgroups in classical groups [4], another investigating intervals in the subgroup lattice of alternating and symmetric groups [3]. John Shareshian [21] suggested some candidates for lattices that may not be representable as intervals in subgroup lattices of finite groups. William DeMeo [10] found representations of all lattices consisting of at most 7 elements, with two exceptions:
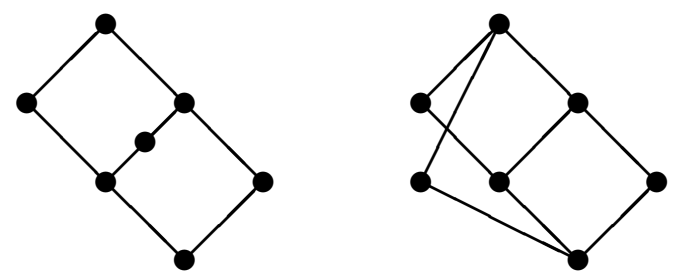

So currently these are the smallest lattices for which no representation as an interval in the subgroup lattice of a finite group is known.

In the present paper our goal was to combine the ideas of Aschbacher, Baddeley, Börner, and Lucchini ([2], [6], [7], [8]) in order to give an accessible proof of the reduction theorem (3.2). The statement of the theorem sligthly differs from Börner's version. On one hand, we have improved case (1) by stating it for all lattices not just for those generated by coatoms. This was made possible by the embedding lemma (3.3). (Börner [8, Lemma 1.1] found a less useful embedding.) On the other hand, our version of (2) is slightly weaker than his, we do not get that $D_{0}$ is a core-free subgroup of $D$. That was achieved by Börner with the help of a more complex lattice than our $L$ and using some additional arguments. The main ideas are also present in the other papers. However, Baddeley [6] gives a reduction that can somewhat alter the lattice to be represented, and Aschbacher [2] formulates the alternative only for a special class of lattices what he calls CD-lattices. Baddeley and Lucchini [7] study lattices of height 2 .

Finally, let us point out some substantial parts from these papers that were used in our presentation. 
- (2.5) and (2.6): $[2,(7.1)]$. On the basis of this remark Aschbacher concentrates on the kernels of the extensions of the homomorphism $\varphi: D_{0} \rightarrow$ $\operatorname{Aut}(T)$. These are subgroups normalized by $D_{0}$ and together with an additional top element form what he calls (the dual of) a signalizer lattice.

- (2.7): Aschbacher's example for the hexagon [2, Example 8.5] is slightly different, he takes $T=A_{5}, D=A_{6} \times A_{6}, D_{0}=\operatorname{diag}\left(A_{5}\right)$.

- (2.13): [8, Lemma 4.10]

- (3.6): Baddeley [6, Definition 3.3] uses a weaker condition, what he calls QP-property. In contrast, Börner's LP-property [8, Definition 2.1] is stronger than ours, making the unnecessary requirement that $y \wedge x=0_{L}$ and $y \vee x=1_{L}$. Aschbacher's A-lattices [2, p. 810] are exactly those what we call strongly non-modular. The conclusion of (3.6) is obtained in each of these papers, and it is emphasized that this corresponds to the notion of quasiprimitive permutation groups.

- Step 1 in the proof of (3.2): [8, Theorems 5.1, 5.2, 5.3]

- Step 2 in the proof of (3.2): [8, Theorem 5.6], [6, part of Theorem 4.9]

- Step 3 in the proof of (3.2): [8, Lemma 5.4]

Acknowledgement. The author has been supported by the National Research, Development and Innovation Fund of Hungary, grant no. 115799.

\section{References}

[1] M. Aschbacher, Finite Group Theory, Cambridge University Press, 1986.

[2] M. Aschbacher, On intervals in subgroup lattices of finite groups, Journal of the American Mathematical Society 21 (2008), 809-830.

[3] M. Aschbacher, Lower signalizer lattices in alternating and symmetric groups, Journal of Group Theory 15 (2012), 151-225.

[4] M. Aschbacher, Overgroups of root groups in classical groups, Memoirs of the American Mathematical Society 241 (2016), no. 1140.

[5] M. Aschbacher and L. L. Scott, Maximal subgroups of finite groups, Journal of Algebra 92 (1985), 44-80.

[6] R. Baddeley, A new approach to the finite lattice representation problem, Periodica Mathematica Hungarica 36 (1998), 17-59.

[7] R. Baddeley and A. Lucchini, On representing finite lattices as intervals in subgroup lattices of finite groups, Journal of Algebra 196 (1997), 1-100.

[8] F. Börner, A remark on the finite lattice representation problem, in Contributions to General Algebra, 11 (I. Chajda et al., eds.) (Verlag Johannes Heyn, Klagenfurt, 1999), 5-38.

[9] P. J. Cameron, Permutation Groups, LMS Student Texts 45, Cambridge University Press, 1999.

[10] W. DeMeo, Congruence lattices of finite algebras, (PhD Thesis, University of Hawaii, 2012) arXiv:1204.4305

[11] G. Grätzer and E. T. Schmidt, Characterizations of congruence lattices of abstract algebras, Acta Scientiarum Mathematicarum (Szeged) 24 (1963), 34-59.

[12] D. Haran, Hilbertian fields under separable algebraic extensions, Inventiones Mathematicae 137 (1999), 113-126. 
[13] B. Huppert, Endliche Gruppen I, Grundlehren der mathematischen Wissenschaften 134 (Springer-Verlag, Berlin-Heidelberg, 1967)

[14] L. G. Kovács, Maximal subgroups in composite finite groups, Journal of Algebra 99 (1986), 114-131.

[15] J. Lafuente, On restricted twisted wreath products of groups, Archiv der Mathematik 43 (1984), 208-209.

[16] M. W. Liebeck, C. E. Praeger and J. Saxl, On the O'Nan-Scott theorem for finite primitive permutation groups, Journal of the Australian Mathematical Society, Ser. A 44 (1988), 389-396.

[17] B. H. Neumann, Twisted wreath product of groups, Archiv der Mathematik 14 (1963), $1-6$.

[18] P. P. Pálfy, Intervals in subgroup lattices of finite groups, in Groups'93 Galway/St Andrews, vol. 2 (C. M. Campbell, T. C. Hurley, E. F. Robertson, S. J. Tobin, J. J. Ward, editors) London Math. Soc. Lecture Notes Ser. 212 (Cambridge University Press, 1995), 482-494.

[19] P. P. Pálfy and P. Pudlák, Congruence lattices of finite algebras and intervals in subgroup lattices of finite groups, Algebra Universalis 11 (1980), 22-27.

[20] L. L. Scott, Representations in characteristic $p$, in Santa Cruz Conference on Finite Groups, Proc. Sympos. Pure Math. 37 (American Mathematical Society, Providence, R.I., 1980), 318-331.

[21] J. Shareshian, Topology of order complexes of intervals in subgroup lattices, Journal of Algebra 268 (2003), 677-686.

[22] M. Suzuki, Group Theory I, Grundlehren der mathematischen Wissenschaften 247 (Springer-Verlag, Berlin-Heidelberg-New York, 1982)

[23] J. Tůma, Intervals in subgroup lattices of infinite groups, Journal of Algebra $\mathbf{1 2 5}$ (1989), 367-399.

[24] Y. Watatani, Lattices of intermediate subfactors, Journal of Functional Analysis 140 (1996), 312-334. 